\title{
Editorial
}

\section{Integrated Approaches to Soil Contamination Monitoring}

\author{
Pantelis Soupios, ${ }^{1}$ Victor Kavvadias, ${ }^{2}$ Katherine Huddersman, ${ }^{3}$ \\ Francesco Sdao, ${ }^{4}$ and Dimitrios Ntarlagiannis ${ }^{5}$ \\ ${ }^{1}$ Technological Educational Institute of Crete, 3 Romanou Chalepa, 73133 Chania, Greece \\ ${ }^{2}$ Hellenic Agricultural Organization-DEMETER, Department of Soil Science of Athens, Institute of Soil and Water Resources, \\ 1 Sofokli Venizelou Street, Lykovrisi, 14123 Attiki, Greece \\ ${ }^{3}$ De Montfort University, The Gateway, Leicester LE1 9BH, UK \\ ${ }^{4}$ School of Engineering, University of Basilicata, Viale dell'Ateneo Lucano 10, Potenza, Italy \\ ${ }^{5}$ Rutgers University, 101 Warren Street, Newark, NJ 07102, USA \\ Correspondence should be addressed to Pantelis Soupios; soupios@staff.teicrete.gr
}

Received 9 May 2016; Accepted 9 May 2016

Copyright (c) 2016 Pantelis Soupios et al. This is an open access article distributed under the Creative Commons Attribution License, which permits unrestricted use, distribution, and reproduction in any medium, provided the original work is properly cited.

Top soil is a very important environmental compartment for many reasons, like it being the medium where plants grow, carbon accumulates, and so forth. But it also represents the "sink" where a wide range of waste materials, in very heterogeneous chemical forms, are disposed of and accumulate. This fact may allow contaminants to move downward the soil profile and reach subsurface and groundwater reservoirs. Agricultural activities can lead to land contamination due to the improper use of pesticides, agrochemicals, fertilizers, conditioners, and several other materials. The problem of contaminated land is exacerbated by industrial activities, including waste disposal and accidental spills that can also contribute to extensive contamination in the near surface environment. Dangerous contaminants can impact the characteristics and productivity of the surface soil as well as the subsurface and valuable natural resources conditions. Soil pollution threats human health, quality of foods, and groundwater but affects also the quality of the air.

Surface and subsurface soil monitoring and characterization can be challenging since chemical analyses at sampling points are local providing an inadequate model of the subsurface. Thus, novel, cost-effective, and multidisciplinary methods are needed to accurately describe surface/subsurface soil contamination whilst monitoring the evolution of the contamination over time producing time-lapse models. Continuous advances on characterization methods (such as automated acquisition systems of subsurface parameters), changes in regulatory standards, and the development of remediation systems further complicate this task.
With this special issue, we aim at bringing together scientists from different disciplines, with research focused on surface soil and subsurface contamination. Furthermore, we want to highlight recent research advances on characterization and monitoring methods and identify the pathways for their implementation to industry, agriculture, and society to encourage their adoption.

The manuscript entitled "Chemical and Physical Characteristics in Uncultivated Soils with Different Lithology in Semiarid Mediterranean Climate" by D. Moraetis et al. aims to identify the chemical and physical characteristics in uncultivated soils derived from different parent materials under semiarid Mediterranean climatic conditions which favored the formation of fragile soils. The authors used PCA analysis to demonstrate the soil quality stage, regarding nutrient availability showing that one of the major physicochemical characteristics such as cation exchange capacity (CEC) is controlled exclusively from mineralogy and not from organic matter. PCA also shows the unusual correlation of $\mathrm{K}^{+}$not only with illite content but also with the OM in fragile soils in Mediterranean climate which is evident in Crete in most of the 54 samples investigated.

The manuscript by C. Noel et al., entitled "Combining Geoelectrical Measurements and $\mathrm{CO}_{2}$ Analyses to Monitor the Enhanced Bioremediation of HydrocarbonContaminated Soils: A Field Implementation," deals with the combined application of Electrical Resistivity (ER) and Induced Polarization (IP) geophysical methods with gas analyses of $\mathrm{CO}_{2}$ concentration and its carbon isotopic ratio, 
to develop a less invasive methodology for monitoring the biodegradation of hydrocarbons. A more conductive and chargeable area which corresponds to the contaminated zone and high $\mathrm{CO}_{2}$ emissions has been measured applying successfully geophysical and chemical methods, respectively. Combining geophysics with gas analyses is therefore a promising tool for in situ monitoring.

The manuscript entitled "Long-Term Dynamics of Urban Soil Pollution with Heavy Metals in Moscow," by N. E. Kosheleva and E. M. Nikiforova, deals with the spatial distributions and concentrations models of the nine heavy metals ( $\mathrm{Zn}, \mathrm{Cd}, \mathrm{Pb}, \mathrm{Cu}, \mathrm{Cr}, \mathrm{Co}, \mathrm{Ni}, \mathrm{Mn}$, and $\mathrm{Cs}$ ) present in top soils located in Moscow territory. Some geochemical anomalies maps, realized using the data collected in 1989, 2005, and 2010, are presented and discussed. The analysis of the geochemical anomalies maps has allowed the identification of areas characterized by different types of pollution phenomena and the evaluation of the environmental risk of the contamination. Finally, the study highlighted a significant increase of the pollution phenomena due to the increase of the heavy metals presence, which could be attributed to the growth of the total amount of industrial and motor transport emissions and change in absorption capacity of the urban soils.

The manuscript "Transport Processes in Porous Media by Self-Potential Method" by V. Giampaolo et al. describes a controlled diffusion/infiltration column experiment aiming at the monitoring of the leakage of a salty water plume by applying time-lapse self-potential (SP) measurements. The measured self-potential values were converted into salt concentration and the sand diffusion $(D)$ and longitudinal dispersivity $(\alpha L)$ values were estimated by modelling the transport equations using the COMSOL Multiphysics.

The manuscript entitled "Aggregate Indices Method in Soil Quality Evaluation Using the Relative Soil Quality Index" by $\mathrm{H}$. N. Pham et al. describes a new approach to assess the soil quality by aggregate indices using the Relative Soil Quality Index (RSQI). The authors applied the RSQI to assess the Soil Environmental Quality of rice intensive cultivation areas through a case study in Haiduong province in 2013. The RSQI is calculated for sampling points in 12 districts and simulated the Soil Environmental Quality on GIS map.

The manuscript entitled "Determination of Tetracycline and Fluoroquinolone Antibiotics at Trace Levels in Sludge and Soil" by M.-V. Salvia et al. describes the development of a sensitive analytical method to determine simultaneously traces of tetracycline and fluoroquinolone antibiotics in sludge and soil, based on a PLE extraction, followed by SPE purification and finally an analysis by LC-MS/MS. The method was successfully applied to the analysis of the target antibiotics in sludge as well as soil that received spreading.

Pantelis Soupios

Victor Kavvadias

Katherine Huddersman

Francesco Sdao

Dimitrios Ntarlagiannis 

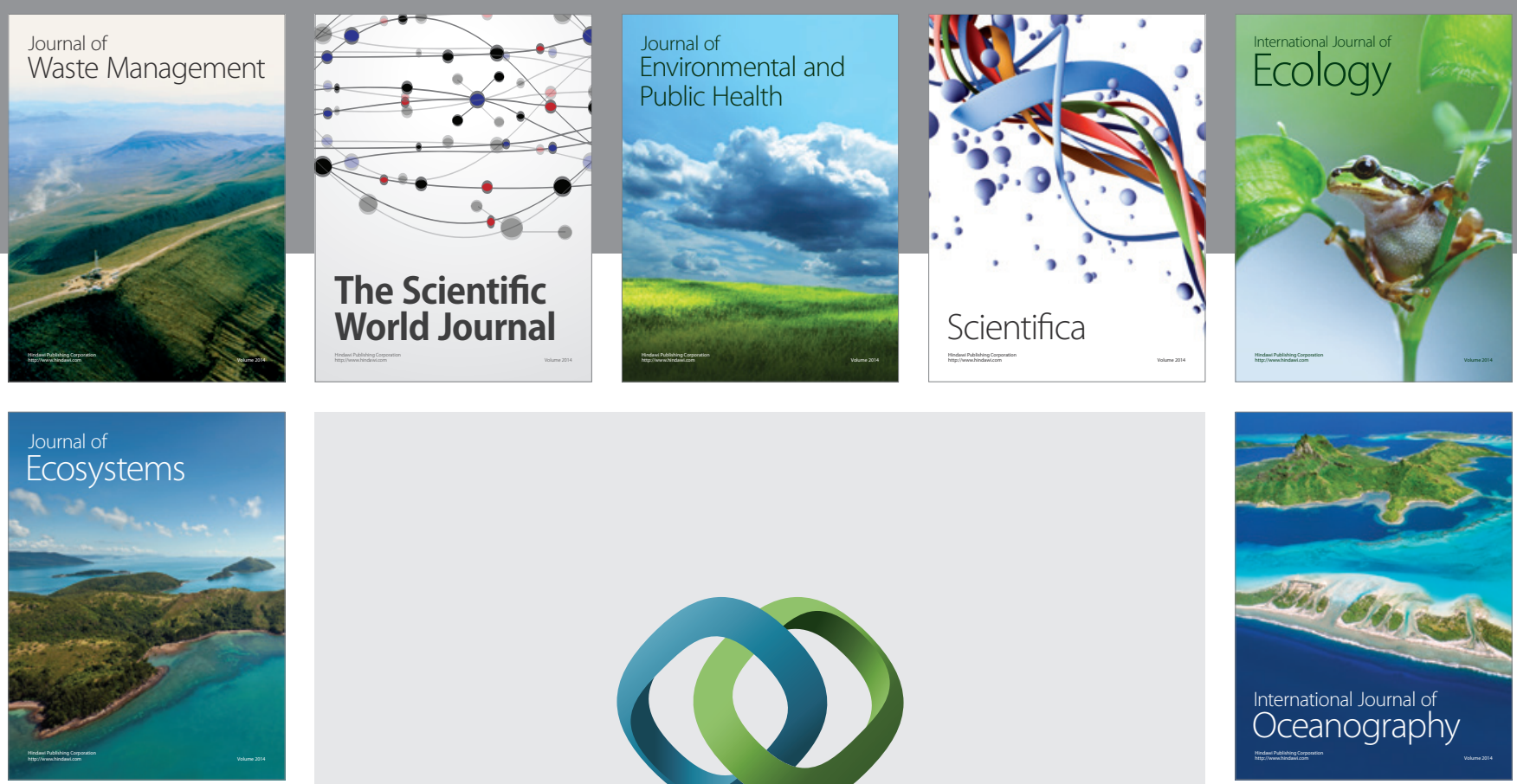

The Scientific World Journal
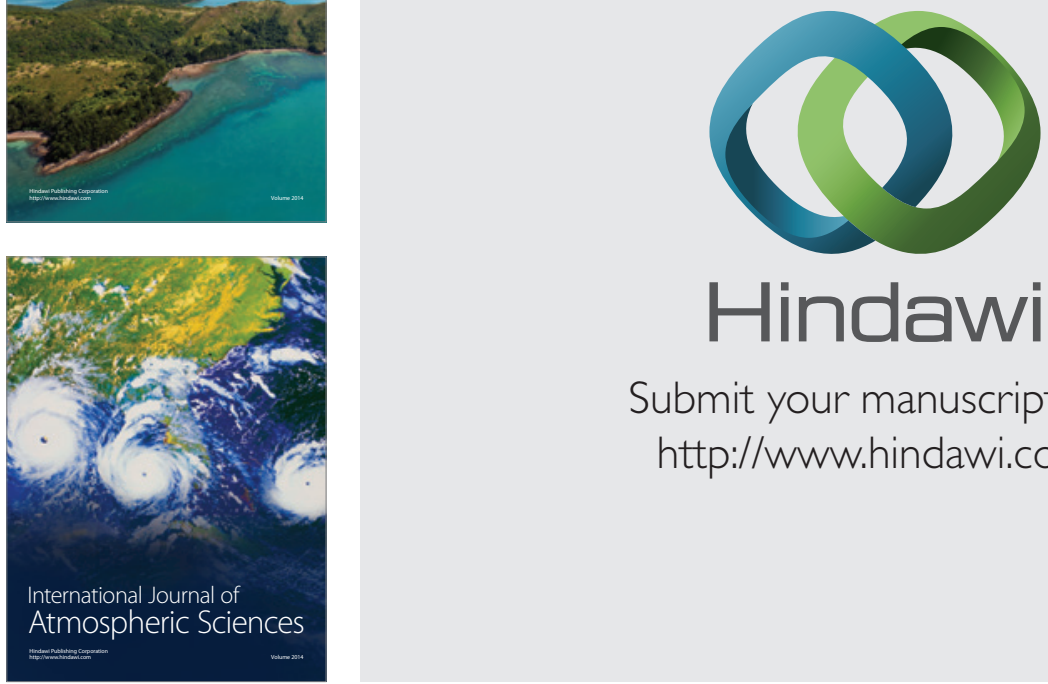

\section{Hindawi}

Submit your manuscripts at

http://www.hindawi.com
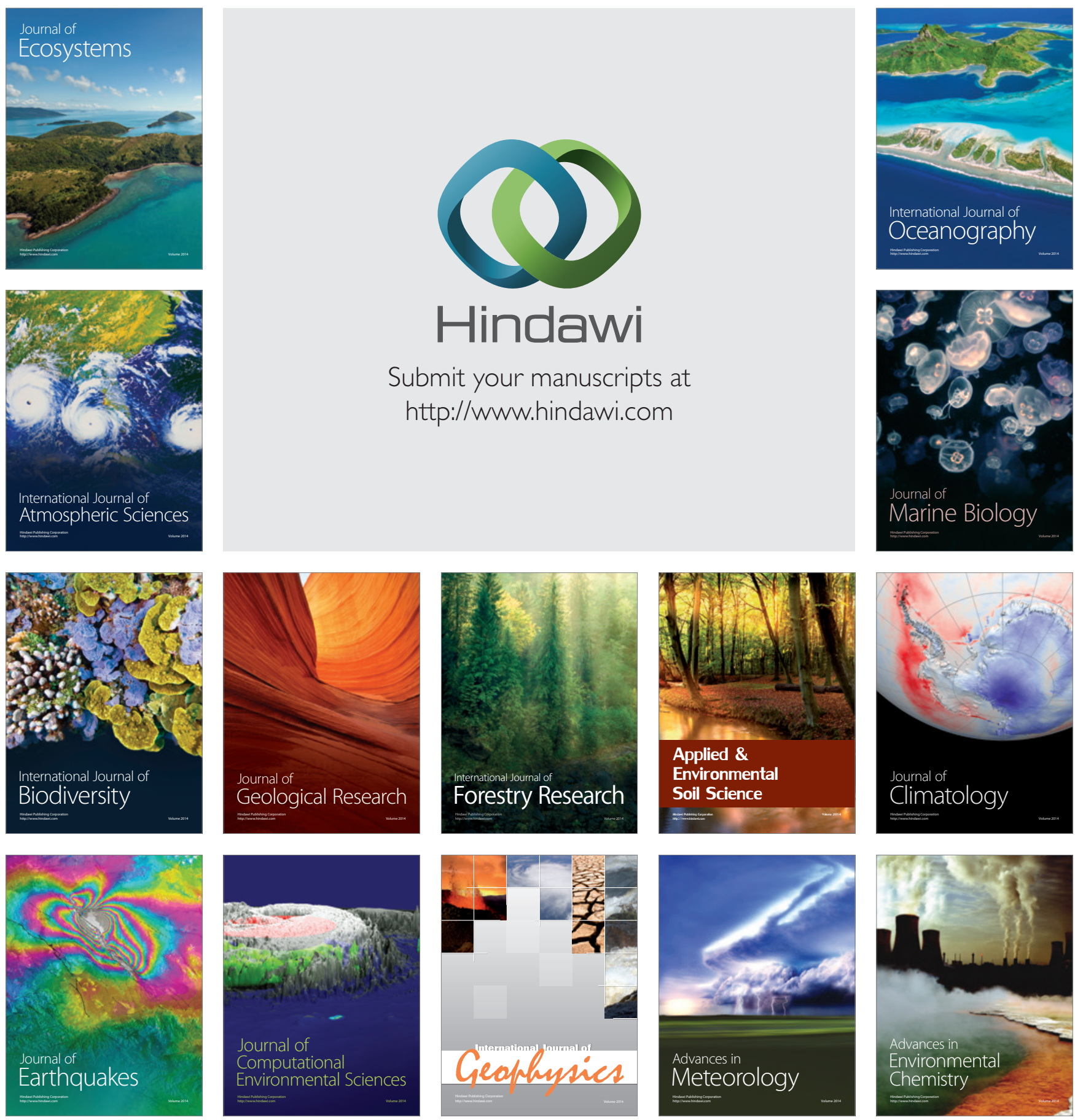